\title{
Invariant metrizability and projective metrizability on Lie groups and homogeneous spaces
}

\author{
Ioan Bucataru, Tamás Milkovszki and Zoltán Muzsnay
}

\begin{abstract}
In this paper we study the invariant metrizability and projective metrizability problems for the special case of the geodesic spray associated to the canonical connection of a Lie group. We prove that such canonical spray is projectively Finsler metrizable if and only if it is Riemann metrizable. This result means that this structure is rigid in the sense that considering left-invariant metrics, the potentially much larger class of projective Finsler metrizable canonical sprays, corresponding to Lie groups, coincides with the class of Riemann metrizable canonical sprays. Generalisation of these results for geodesic orbit spaces are given.
\end{abstract}

2000 Mathematics Subject Classification: 53B05, 53B40, 70H03, 70H30, 70F17.

Key words and phrases: Euler-Lagrange equation; geodesics; metrizability and projective metrizability; Lie group, homogeneous space; geodesic orbit structure.

\section{Introduction}

Lie groups represent a well developed theory of continuous symmetries of mathematical structures, and it is an indispensable tool for modern theoretical physics. The algebraic and differential structures allow us to consider a class of natural objects which have been extensively investigated for over 100 years. Among these, one of the most interesting, from the differential geometric point of view, is the canonical geodesic structure. It consists of the family of curves given by the 1-dimensional subgroups of a Lie group and their left translated images. The quadratic second order differential equation (SODE) associated to this geodesic structure is called the canonical SODE and the vector field corresponding to the geodesic flow is called the canonical spray of a Lie group.

In this paper we investigate the Riemann and Finsler metrizability and projective metrizability of the canonical spray of a Lie group. A spray is called Riemann (resp. Finsler) metrizable, if there exists a Riemann (resp. Finsler) metric such that its geodesics are the geodesics of the spray. For the more general projective metrizability problem, one seeks a Riemann (resp. Finsler) metric whose geodesics coincide with the geodesics of the spray, up to an orientation preserving reparameterization. Recently, several papers have appeared on the metrizability and projective metrizability problems [3, 5, 8, 13. These articles show that the metrizability and projective metrizability problems are very complex, and even in low dimensional cases, the complete classification is very difficult. Concerning Lie groups, G. Thompson and his co-workers investigated the inverse problem of Lagrangian dynamics for the canonical spray in a series of papers [10, 11, 18, 22, 23]. The problem of the existence of a left-invariant variational principle for the canonical spray was considered in [14, 16], and for invariant second-order differential equations, using the Helmholtz conditions, in [7]. 
The notion of a Finsler metric is a generalisation of the notion of a Riemannian metric, as Shiing-Shen Chern says "Finsler geometry is just Riemannian geometry without the quadratic restriction", [6]. Therefore, every Riemann metrizable spray (necessarily quadratic) is trivially Finsler metrizable. The converse, in general, is not true; there are Finsler metrizable sprays (necessarily non-quadratic) which are not Riemann metrizable. Even so, in the category of quadratic sprays, Szabó's theorem [20] states that the two notions of Finsler metrizability and Riemann metrizability coincide. The projective Finsler metrizability, however, is essentially different from projective Riemann metrizability, even in the case of quadratic sprays. This follows since a quadratic spray can be projectively equivalent, using a non-linear projective factor, to a non-quadratic one and hence cannot be Riemann metrizable. Therefore, the category of projective Finsler metrizable sprays is generally strictly larger then the category of Riemann metrizable sprays, even for quadratic sprays.

The goal of this paper is to investigate the relationship between invariant metrizability, and the invariant projective metrizability of the canonical spray of a Lie group. In the case of the invariant metrizability problem, we ask if there exists a left-invariant Riemann (resp. Finsler) metric, such that its geodesics are the geodesics of the canonical spray. In the case of the invariant projective metrizability problem, we ask if there exists a left-invariant Riemann (resp. Finsler) metric, such that its geodesics are projectively equivalent to the geodesics of the canonical spray. We prove that the canonical connection of a Lie group is invariant projective Finsler metrizable if and only if it is invariant Riemann metrizable. This result shows that the structure is rigid in the sense that by considering left-invariant metrics, the potentially much larger class of projective Finsler metrizable canonical sprays, corresponding to Lie groups, coincides with the class of Riemann metrizable canonical sprays.

We consider also homogeneous spaces $G / H$ with a special geodesic structure. A left invariant geodesic structure on $G / H$ is called geodesic orbit structure (g.o. structure), if the geodesics can be derived as orbits of 1-parameter subgroups of $G$. In V.I. Arnold's terminology these curves are called "relative equilibria" 2]. We prove that a g.o. structure is projectively invariant Riemann (resp. Finsler) metrizable if and only if it is invariant Riemann (resp. Finsler) metrizable. In the quadratic case we also obtain the rigidity property: the class of projective Finsler metrizable and Riemann metrizable g.o. sprays coincide.

\section{Differential geometric background of the inverse problem of the calculus of variations}

In this section we present the basic objects and tools required for our investigation. More details can be found in [5, 12].

\section{SODEs, sprays and associated connection}

Let $M$ be a smooth, finite dimensional manifold, $T M$ its tangent bundle and $\pi$ the natural projection. The map $J: T T M \rightarrow T T M$ denotes the canonical vertical endomorphism and $C \in \mathfrak{X}(T M)$ the Liouville vector field. If $x=\left(x^{i}\right)$ is a local coordinate system on $M$ and $(x, y)=\left(x^{i}, y^{i}\right)$ is the induced coordinate system on $T M$, we then have that $J=d x^{i} \otimes \frac{\partial}{\partial y^{i}}$, and $C=y^{i} \frac{\partial}{\partial y^{i}}$. Using the vector field $C$ and Euler's theorem on homogeneous functions, a Lagrangian $L: T M \rightarrow \mathbb{R}$ is an $l$-homogeneous function in the $y$ variable if and only if

$$
C F=l \cdot F \text {. }
$$


A spray is a vector field $S$ on $T M \backslash\{0\}$ satisfying the relations $J S=C$ and $[C, S]=S$. The coordinate representation of a spray $S$ takes the form

$$
S=y^{i} \frac{\partial}{\partial x^{i}}+f^{i}(x, y) \frac{\partial}{\partial y^{i}},
$$

where the functions $f^{i}(x, y)$ are homogeneous of degree 2 in $y . S$ is called quadratic, if $f^{i}(x, y)$ are quadratic in $y$. The geodesics of a spray $S$ are the curves $\gamma: I \rightarrow M$ such that $\dot{\gamma}$ is an integral curve of $S$, that is $S \circ \dot{\gamma}=\ddot{\gamma}$. The curve $\gamma$ is a geodesic of (2.2) if and only if it is a solution of the SODE: $\ddot{x}^{i}=f^{i}(x, \dot{x}), i=1, \ldots, n$. Two family of curves are projectively equivalent, if they coincide up to an orientation preserving reparameterization. In this spirit we also call two SODEs (or sprays) projectively equivalent if their solutions (as parametrised curves) are projectively equivalent. It is easy to show that two sprays $S$ and $\bar{S}$ are projectively equivalent if and only if there exists a 1-homogeneous function $\mathcal{P}=\mathcal{P}(x, y)$ such that $\bar{S}=S-2 \mathcal{P} C$.

A non-linear connection on $M$ is a type (1-1) tensor field $\Gamma$ on $T M$ such that $J \Gamma=J$ and $\Gamma J=-J$. If $\Gamma$ is a non-linear connection, then $\Gamma^{2}=i d_{T T M}$ and the eigenspace corresponding to the eigenvalue -1 is the vertical space. The eigenspace $H$ corresponding to the eigenvalue +1 is called the horizontal space. In the sequel we will write $h=\frac{1}{2}(I+\Gamma)$ and $v=\frac{1}{2}(I-\Gamma)$, for the horizontal and vertical projectors. If $S$ is a spray, then $\Gamma=[J, S]$ is a non-linear connection which is called the canonical connection associated to $S$.

\section{The Euler-Lagrange ODE and PDE}

It is well known that if a Lagrangian $L: T M \rightarrow \mathbb{R}$ is regular, that is $\operatorname{det}\left(\frac{\partial^{2} L}{\partial y^{i} \partial y^{j}}\right) \neq 0$, then the 2 -form $\Omega_{L}:=d d_{J} L$ has maximal rank. For a regular Lagrangian $L$, homogeneous of degree 2 , the vector field $S$ on $T M$ defined by the equation

$$
i_{S} \Omega_{L}+d\left(d_{C} L-L\right)=0
$$

(where $d_{C} L$ denotes the Lie derivative of $L$ with respect to $C$ ) is a spray, and the geodesics of $S$ are the solutions of the Euler-Lagrange ODE.

Let us fix a spray $S$ on the manifold $M$. Then, to every Lagrangian $L$, a scalar 1 -form $\omega_{L}=i_{S} \Omega_{L}+d\left(d_{C} L-L\right)$, called the Euler-Lagrange form can be associated. Then the Euler-Lagrange equation can be written as

$$
\omega_{L}=0 .
$$

The equation (2.4) holds if and only if the geodesics of the spray $S$ are the solutions of the Euler-Lagrange equation associated to $L$. We remark, that when $S$ is given, (2.4) is a system of second order PDE on $L$ called the Euler-Lagrange PDE.

\section{Metrizability and projective metrizability}

The metrizability and projective metrizability problems, for a given spray $S$, can be formulated as follows.

Definition 3.1. A spray $S$ is

1) Riemann (resp. Finsler) metrizable, if there exists a Riemann (resp. Finsler) metric whose geodesics coincide with the geodesics of $S$. 
2) projective Riemann (resp. Finsler) metrizable, if there exists a Riemann (resp. Finsler) metric whose geodesics are projectively equivalent with the geodesics of $S$.

Both the metrizability and projective metrizability problems can be formulated in terms of a system of partial differential equations which is composed of the appropriate homogeneity condition and the Euler-Lagrange PDE equations on the energy function.

Proposition 3.2. A spray $S$ on a manifold $M$ is

1) Riemann (resp. Finsler) metrizable if and only if there exists a quadratic (resp. homogeneous of degree 2) function $E: T M \rightarrow \mathbb{R}$, such that the matrix field $\left(\frac{\partial^{2} E}{\partial y^{i} \partial y^{j}}\right)$ is positive definite on $T M \backslash\{0\}$ and the equation 2.4) is satisfied with $L:=E$.

2) projectively Riemann (resp. Finsler) metrizable if and only if there exists a quadratic (resp. homogeneous of degree 2) function $\widehat{E}: T M \rightarrow \mathbb{R}$, such that the matrix field $\left(\frac{\partial^{2} \widehat{E}}{\partial y^{2} \partial y^{3}}\right)$ is positive definite on $T M \backslash\{0\}$ and the equation 2.4 is satisfied with $L:=$ $\sqrt{2 \widehat{E}}$.

Proof. For 1) we remark that the Riemann (resp. Finsler) metric $g=g_{i j} d x^{i} \otimes d x^{j}$ exists if and only if the associated energy function $E=g_{i j} y^{i} y^{j} / 2$ exists. Thus a function $E$ is the Riemann (resp. Finsler) energy function corresponding to $S$ if and only if it satisfies the conditions formulated in point 1) of the proposition.

For 2) we note that the spray $S$ is projectively Riemann (resp. Finsler) metrizable if and only if there exists a Riemann (resp. Finsler) metrizable spray $\widehat{S}$ which is projectively equivalent to $S$. In that case there exists a function $\mathcal{P}$, homogeneous of degree 1 , such that $\widehat{S}=S-2 \mathcal{P} C$. Let us denote by $\widehat{E}=\widehat{g}_{i j} y^{i} y^{j} / 2$ the energy function of $\widehat{S}$ and let $\widehat{F}=\sqrt{2 \widehat{E}}$ be the associated Finsler function. It is well known that $\widehat{F}$ is invariant with respect to the parallel translation associated to the canonical non-linear connection $\widehat{\Gamma}$ and therefore we have $d_{\widehat{h}} \widehat{F}=0$ where $\widehat{h}=h-\mathcal{P} J-d_{J} \mathcal{P} \otimes C$ (see [4, section 4). Using the homogeneity of degree 1 of the $\widehat{F}$, we get

$$
0=d_{\widehat{h}} \widehat{F}=d_{h} \widehat{F}-d_{J}(\mathcal{P} \widehat{F}) .
$$

Substituting $S$ into the above formula and using the homogeneity of degree 1 of $\mathcal{P}$ and $\widehat{F}$ we get $d_{\widehat{h}} \widehat{F}(S)=S \widehat{F}-2 \mathcal{P} \widehat{F}=0$. It follows that the projective factor is given by

$$
\mathcal{P}=\frac{1}{2 \widehat{F}} S \widehat{F} .
$$

Replacing $\mathcal{P}$ in formula (3.1) and using the Frölicher-Nijenhuis formalism we get

$$
0=2 d_{h} \widehat{F}-d_{J}\left(\frac{1}{\widehat{F}} d_{S} \widehat{F} \widehat{F}\right)=d_{\Gamma+I} \widehat{F}-d_{J} d_{S} \widehat{F}=d_{[J, S]} \widehat{F}+d \widehat{F}-d_{J} d_{S} \widehat{F}=-i_{S} d d_{J} \widehat{F} .
$$

On the other hand, since $\widehat{F}$ is a function homogeneous of degree 1 , we have $C \widehat{F}=\widehat{F}$ and $d d_{C} \widehat{F}-d \widehat{F}=0$. Therefore, the Euler-Lagrange form is $\omega_{\widehat{F}}=i_{S} d d_{J} \widehat{F}+d d_{C} \widehat{F}-d \widehat{F}=i_{S} d d_{J} \widehat{F}$. Comparing this with (3.3) we get that $\omega_{\widehat{F}}=0$. This shows that the conditions of 2) are necessary conditions.

Conversely, let us suppose that the function $\widehat{E}$ satisfies the conditions of 2). If we define the projective factor $\mathcal{P}$ by using formula (3.2), then the spray $\widehat{S}=S-2 \mathcal{P} C$ is projectively equivalent to the spray $S$. It is not difficult to show that the spray associated to $\widehat{E}$ is given by $\widehat{S}$.

We note, that a coordinate version of 2) was first proved by A. Rapcsák in [19] and a coordinate free version of this the statement was given by J. Szilasi and Sz. Vattamány in [21]. Here we have presented a different approach. 
Remark 3.3. For a given spray $S$, the second order partial differential equation $i_{S} d d_{J} F=$ 0 is called the Rapcsák equation. As we have seen in equation (3.3), for functions homogeneous of degree 1, the Rapcsák equation is equivalent to the Euler-Lagrange equation.

Proposition 3.4. Let $S$ be a spray and $L$ be a Lagrangian. If $L$ is a first integral for $S$, then we have

$$
\omega_{L}=0 \quad \Leftrightarrow \quad d_{h} L=0
$$

Proof. Consider $L$ a first integral for $S$, that is $S(L)=d_{S} L=0$. Then, using the FrölicherNijenhuis calculus, we get

$$
\begin{aligned}
\omega_{L} & =i_{S} d d_{J} L+d d_{C} L-d L=d_{S} d_{J} L-d i_{S} d_{J} L+d d_{C} L-d L \\
& =d_{[S, J]} L+d_{J} d_{S} L-d\left(d_{J S}-d_{J} i_{S}+i_{[J, S]} L+d d_{C} L-d L\right. \\
& =-d_{\Gamma} L-d L=-d_{\Gamma+I} L=-2 d_{h} L,
\end{aligned}
$$

which shows the equivalence of the two conditions of (3.4).

Corollary 3.5. Let $S$ be a spray, let $L$ be a non-zero first integral for $S$ and $f$ a smooth non-vanishing function on $\mathbb{R}$ with non-vanishing derivative. Then, L satisfies the EulerLagrange equation (2.4), associated to $S$, if and only if $f(L)$ satisfies the Euler-Lagrange equation (2.4), associated to $S$

Proof. Using Theorem 3.4 we know that equation $\omega_{L}=0$ is equivalent to $d_{h} L=0$ and equation $\omega_{f(L)}=0$ is equivalent to $d_{h}(f(L))=0$. Moreover, since $f^{\prime}(L) \neq 0$ and

$$
d_{h}(f(L))=f^{\prime}(L) d_{h} L,
$$

we have $d_{h}(f(L))=0$ if and only if $d_{h} L=0$ holds.

We will use Corollary 3.5 for the particular case when $f(t)=t^{k}$. This general form of Corollary 3.5 corresponds to [1, Proposition 3.2] and it was suggested to us by am anonymous reviewer, to whom we express our thanks.

\section{Invariant metrizability and projective metrizability of the canonical flow of Lie groups}

Let $G$ be a finite dimensional Lie group. We denote by $\lambda_{g}: G \rightarrow G$ the left translation of $G$ defined by $\lambda_{g}(\hat{g})=g \hat{g}$. Let $x=\left(x^{1}, \ldots, x^{n}\right)$ be a coordinate system on $G$ and $(x, y)$ be the usual associated standard coordinate system on $T G$ where $y=\left(y^{1}, \ldots, y^{n}\right)$ with $y^{i}=d x^{i}$. We will be interested in investigating left invariant structures on $T G$. It is thus more convenient to introduce a kind of "semi-invariant" coordinate system using the left trivialisation $T G \cong G \times \mathfrak{g}$. Indeed, for every $g \in G$, the tangent space $T_{g} G$ is isomorphic to $\mathfrak{g}$ by the tangent map of the left translation $\left(\lambda_{g}^{-1}\right)_{*}: T_{g} G \rightarrow \mathfrak{g}=T_{e} G$. Therefore, one can introduce a left invariant $\mathfrak{g}$-valued differential form $\theta: T G \rightarrow \mathfrak{g}$, known as the MaurerCartan form, defined by $\theta=\left(\lambda_{g}^{-1}\right)_{*} d g$. The corresponding semi-invariant coordinate system is given by $\left(x^{i}, \alpha^{i}\right)$ where $\alpha^{i}=\left(\lambda_{x, *}^{-1}\right)_{j}^{i} d x^{j}$. The left invariant coordinate system $(x, \alpha)$ induces coordinates on the second tangent bundle TTG which will be denoted by

$$
\left.\left(x^{i}, \alpha^{i}, X^{i}, A^{i}\right) \simeq X^{i} \frac{\partial}{\partial x^{i}}\right|_{(x, \alpha)}+\left.A^{i} \frac{\partial}{\partial \alpha^{i}}\right|_{(x, \alpha)},
$$


We note that, by using a simplified notation

$$
\left(x^{i}, \alpha^{i}, X^{i}, A^{i}\right) \simeq(x, \alpha, X, A),
$$

the coordinates $x=\left(x^{i}\right)$, and therefore the coordinates $X=\left(X^{i}\right)$ are not left invariant, but $\alpha=\left(\alpha^{i}\right)$ and therefore the corresponding $A=\left(A^{i}\right)$, are: the left translation by a group element $g$ induces on TTG the following action: $\left(\lambda_{g}\right)_{* *}(x, \alpha, X, A)=\left(\lambda_{g} x, \alpha, \lambda_{g *} X, A\right)$.

Definition 4.1. A vector field $X \in \mathfrak{X}(G)$ is called left invariant, if $\left(\lambda_{g}\right)_{*} X=X$ for every $g \in G$. Similarly, a function $L: T G \rightarrow \mathbb{R}$ is called left invariant, if $L \circ \lambda_{g *}=L, \forall g \in G$.

Using the semi-invariant coordinate system $\left(x^{i}, \alpha^{i}\right)$, the function $L: T G \rightarrow \mathbb{R}$ is left invariant if and only if its value does not depend on the $x$-coordinates, that is

$$
\frac{\partial L}{\partial x^{i}} \equiv 0, \quad i=1, \ldots, n .
$$

Definition 4.2. The canonical geodesic structure on a Lie group $G$ is given by the 1parameter subgroups and their left (or right) translated images. The canonical SODE of $G$ is the SODE corresponding to the canonical flow.

The main geometric objects associated to the canonical flow (spray, horizontal and vertical projections etc.) were calculated in [16] and [18]. Here we just present the essential results needed for our purpose. More about the computation of these objects can be found in the above mentioned papers.

Using a $G \rightarrow G L(n, \mathbb{R}), x \rightarrow M_{x}$ matrix representation, the canonical SODE can be described by

$$
\ddot{M}_{t}=\dot{M}_{t} M_{t}^{-1} \dot{M}_{t}
$$

where we denote $M_{t}:=M_{x_{t}}$. The canonical spray of a Lie group $G$, in the semi-invariant coordinate system $(x, \alpha)$, using the simplified notation (4.1), is given by

$$
S_{(x, \alpha)}=\left.\lambda_{x} \alpha \frac{\partial}{\partial x}\right|_{(x, \alpha)} .
$$

The vertical and horizontal projectors are defined as follows. For every $(x, \alpha) \in T G$ we have

$$
\begin{aligned}
v\left(\left.\lambda_{x} a \frac{\partial}{\partial x}\right|_{(x, \alpha)}+\left.b \frac{\partial}{\partial \alpha}\right|_{(x, \alpha)}\right) & =\left.\left(\frac{1}{2}[a, \alpha]+b\right) \frac{\partial}{\partial \alpha}\right|_{(x, \alpha)}, \\
h\left(\left.\lambda_{x} a \frac{\partial}{\partial x}\right|_{(x, \alpha)}+\left.b \frac{\partial}{\partial \alpha}\right|_{(x, \alpha)}\right) & =\left.\lambda_{x} a \frac{\partial}{\partial x}\right|_{(x, \alpha)}-\left.\frac{1}{2}[a, \alpha] \frac{\partial}{\partial \alpha}\right|_{(x, \alpha)} .
\end{aligned}
$$

We have the following

Proposition 4.3. (Canonical invariant Euler-Lagrange system)

A Lagrangian $L: T G \rightarrow \mathbb{R}$ is a left-invariant solution to the Euler-Lagrange equation associated to the canonical spray of the Lie group $G$, if and only if the system

$$
\begin{aligned}
\frac{\partial L}{\partial x^{i}} & =0, \quad i=1, \ldots, n \\
{[a, \alpha]^{i} \frac{\partial L}{\partial \alpha^{i}} } & =0, \quad \forall a \in \mathfrak{g},
\end{aligned}
$$

is satisfied. 
Proof. The equation (4.7) expresses the left invariant property of $L$. Moreover, from the local expression (4.4) of the canonical spray $S$ it is clear, that if the Lagrangian $L$ is left invariant, then it is also a first integral of $S$, which means that

$$
d_{S} L=0 .
$$

Using Proposition 3.4, we get that the Euler-Lagrange PDE is satisfied if and only if $d_{h} L=0$. Using (4.6) we have

$$
d_{h} L=0 \quad \Leftrightarrow \quad \lambda_{x} a \frac{\partial L}{\partial x}-\frac{1}{2}[a, \alpha] \frac{\partial L}{\partial \alpha}=0, \quad a \in \mathfrak{g} .
$$

and from the left invariant property we get the later is identically zero if and only if the equations (4.8) are satisfied.

The following statement holds.

Proposition 4.4. The canonical spray $S$ of a Lie group is left invariant projectively Riemann (resp. Finsler) metrizable if and only if it is left invariant Riemann (resp. Finsler) metrizable.

Proof. It is clear that if $S$ is Riemann (resp. Finsler) metrizable, then it is also projectively Riemann (resp. Finsler) metrizable. Conversely, let us suppose that $S$ is projectively Riemann (resp. Finsler) metrizable. Then, according to Proposition 3.2, there exists a left invariant (quadratic, resp. homogeneous of degree 2) function $\widehat{E}: T G \rightarrow \mathbb{R}$ such that the matrix field $\left(\frac{\partial^{2} \widehat{E}}{\partial y^{2} \partial y^{j}}\right)$ is positive definite on $T M \backslash\{0\}$ and $\widehat{F}:=\sqrt{2 \widehat{E}}$ satisfies the Euler-Lagrange PDE associated to $S$. Because of the left invariance condition, we have $d_{S} L=0$ and, using Corollary 3.5, we get that $\widehat{E}:=\frac{1}{2}(\widehat{F})^{2}$ is also a solution of the Euler-Lagrange PDE associated to $S$. Then, according to Proposition 3.2 , the given $\widehat{E}$ is the energy function of a Riemann (resp. Finsler) metric which implies that $S$ is Riemann (resp. Finsler) metrizable.

We have the following result.

Theorem 4.5. The canonical spray of a Lie group is left invariant projectively Finsler metrizable if and only if it is left invariant Riemann metrizable.

Proof. In one direction the statement is trivial. If the canonical spray is Riemann metrizable, then it is trivially Finsler metrizable and also projectively Finsler metrizable.

Let us consider the converse statement, and suppose that the canonical spray $S$ is projectively Finsler metrizable. Then, according to Proposition 4.4, it is also Finsler metrizable. Since $S$ is quadratic, it follows that the associated connection is linear. Hence, the Finsler metrizability induces the existence of a Berwald metric on the Lie group. Using Szabó's theorem which states that for every Berwald metric there exists a Riemannian metric such that the geodesics of the Berwald and Riemannian metrics are the same (cf. [20]), we get that the canonical spray is Riemann metrizable.

Using Proposition 4.3 we obtain the following.

Corollary 4.6. The canonical spray of a Lie group G is left invariant (Riemann, Finsler, projectively Riemann or projectively Finsler) metrizable if and only if there exists a scalar product $\langle$,$\rangle on \mathfrak{g}$ such that

$$
\langle[a, \alpha], \alpha\rangle=0
$$

for every $a, \alpha \in \mathfrak{g}$. 
Proof. An invariant Riemannian metric induces a scalar product $\langle$,$\rangle on \mathfrak{g}$. Using the coordinate system $(x, \alpha)$ on $T G \simeq G \times \mathfrak{g}$, the associated energy function is given by $E: G \times \mathfrak{g} \rightarrow \mathbb{R}$, where $E(x, \alpha)=\langle\alpha, \alpha\rangle$. The Euler-Lagrange equation (4.8) then implies (4.10).

Remark 4.7. We want to draw attention to few interesting phenomena. First of all, although the canonical spray of a Lie group is a very natural object, it is not true that it is always metrizable. In [10] there are several examples of Lie groups and Lie algebras where the canonical spray is non metrizable.

Secondly, despite the fact that the canonical spray is left (and also right) invariant, and the Euler-Lagrange equation inherits the symmetries of the Lagrangian, it is not true that the "metrizability" property means automatically "metrizability by a left invariant metric".

Indeed, for example the 3-dimensional Heisenberg group $\mathbb{H}_{3}$ is not metrizable or projectively metrizable with an invariant Riemann (or Finsler) metric [16]. However, since the curvature tensor vanishes identically, the canonical spray is metrizable. The corresponding (non invariant) Riemannian metric is given by $g=d x^{2}+d y^{2}+\left(d z-\frac{y}{2} d x-\frac{x}{2} d y\right)^{2}$, (see [10]).

Theorem 4.5 shows that the geometric structure associated to the canonical spray of a Lie group has a certain rigidity property. The potentially much larger class of Lie groups where the canonical spray is projectively equivalent to a Finsler geodesic structure, actually coincides with the class of invariant Riemann metrizable sprays. We note that this property relies heavily on the fact that, 1) the canonical spray is quadratic, and 2) the Lie derivative of a left invariant Lagrange function on $G$, with respect to the canonical spray, is identically zero. The second property is not true in general for an arbitrary left invariant spray. However, interesting generalisation can be obtained by considering the class of homogeneous spaces. We consider this in the next section.

\section{Invariant metrizability and projective metrizability of a geodesic orbit structure of homogeneous spaces}

Let $M$ be a connected differentiable manifold on which the Lie transformation group $G$ acts transitively. Let us fix an origin $o \in M$ and denote by $H$ the stabiliser of $o \in M$ in the group $G$ and by $\pi: G \rightarrow G / H$ the projection map. As usual we call $H$ the isotropy group of the homogeneous space $G / H$. Then $M$ is isomorphic to the factor space $G / H$ with origin $H$ and its tangent space at $o \in M$ is isomorphic to $\mathfrak{g} / \mathfrak{h}$, where $\mathfrak{g}$ and $\mathfrak{h}$ are the Lie-algebras of the Lie groups $G$ and $H$ respectively. The action of $G$ on $M$ is determined by the map

$$
\lambda:(g, m) \mapsto \lambda_{g} m=g \cdot m: G \times M \rightarrow M .
$$

Geodesic structures, sprays, metrics, Lagrangians on $M$ are called invariant, if they are invariant with respect to the action of $G$. It is clear, that invariant sprays, metrics, and Lagrangians can be characterised by their values on $T_{o} M$.

In the most interesting cases, the algebraic structures and invariant geometric structures are intimately related: the geodesics are the image of the 1-parameter subgroups of the group $G$ and their left translated images. More precisely we have the following

Definition 5.1. A geodesic $\gamma(t)$, emanating from the origin $o \in M$, is called homogeneous, if there exists $X_{\gamma} \in \mathfrak{g}$, such that $\gamma(t)$ is the orbit of the 1-parameter subgroup $\left\{\exp t X_{\gamma}, t \in\right.$ $\mathbb{R}\}$ of $G$, that is

$$
\gamma(t)=\lambda_{\exp t X_{\gamma}} o=\left(\exp t X_{\gamma}\right) \cdot o
$$


The Lie algebra element $X_{\gamma} \in \mathfrak{g}$ is called the geodesic vector associated to the direction $\dot{\gamma}(0) \in T_{o} M$.

Definition 5.2. A left invariant geodesic structure is called geodesic orbit structure (g.o. structure), if any geodesic $\gamma(t)$ emanating from the origin $o \in M$ is homogeneous (in the sense of Definition [5.1). A spray is called geodesic orbit spray (g.o. spray), if it corresponds to a g.o. structure.

Homogeneous geodesics are called in V.I. Arnold's terminology "relative equilibria" [2].

Definition 5.3. A map $\sigma: T_{o} M \rightarrow \mathfrak{g}$ is called a homogeneous lift 1 if the following conditions are satisfied:

1. $\pi_{*} \circ \sigma=i d_{T_{o} M}$.

2. $\sigma$ is 1-homogeneous, that is $\sigma(\kappa \cdot v)=\kappa \sigma(v)$, for every $v \in T_{o} M$ and $\kappa \in \mathbb{R}$.

3. $\sigma$ is $\operatorname{Ad}(H)$-invariant, that is $\sigma\left(\lambda_{h *} v\right)=\operatorname{Ad}_{h} \sigma(v)$ for all $h \in H$ and $v \in T_{o} M$.

The homogeneous lift $\sigma$ is called $\mathcal{C}^{\infty}$-differentiable if it is continuous on $T_{o} M$ and $\mathcal{C}^{\infty}$ differentiable on $T_{o} M \backslash\{0\}$.

It is clear that any g.o. spray determines a $\mathcal{C}^{\infty}$-differentiable homogeneous lift by associating to $v \in T_{o} M$ its geodesic vector $X=\sigma(v)$ and vice versa, every homogeneous lift determines a g.o. spray by left translations.

Lemma 5.4. Invariant functions are constant along the geodesics of a g.o. spray.

Proof. Because of the invariance, it is enough to show this property for geodesics emanating from the origin $o \in M$. Let $S$ be a geodesic orbit spray on a homogeneous space $M=G / H$. For a geodesic $\gamma(t)$ emanating from the origin $o \in M$ there exists a geodesic vector $X_{\gamma} \in \mathfrak{g}$ such that we have (5.1). Using the 1-parameter subgroup property we can write

$$
\gamma\left(t_{0}+t\right)=\lambda_{\exp \left(t_{0}+t\right) X_{\gamma}} O=\lambda_{\exp t_{0} X_{\gamma}} \lambda_{\exp t X_{\gamma}} O
$$

and therefore we have

$$
\dot{\gamma}\left(t_{0}\right)=\left.\frac{d}{d t}\right|_{t=0} \gamma\left(t_{0}+t\right)=\left.\frac{d}{d t}\right|_{t=0} \lambda_{\exp t_{0} X_{\gamma}} \lambda_{\exp t X_{\gamma}} O=\left(\lambda_{\exp t_{0} X_{\gamma}}\right)_{*} \dot{\gamma}(0) .
$$

Let $L: T M \rightarrow \mathbb{R}$ be an invariant function. For every $p \in M, v \in T_{p} M$ and $g \in G$ we have $L(v)=L\left(\lambda_{g, *} v\right)$. Applying this with $v=\dot{\gamma}(0)$ and $g=\exp t_{0} X_{\gamma}$ we get from (5.3) that $L\left(\dot{\gamma}\left(t_{0}\right)\right)=L(\dot{\gamma}(0))$ that is $L$ is constant on the geodesics.

Using Lemma 5.4 we can obtain the following generalisation of the Proposition 4.4.

Proposition 5.5. A g.o. spray is projectively Riemann (resp. Finsler) invariant metrizable if and only if it is invariant Riemann (resp. Finsler) metrizable.

Proof. Let $S$ be a g.o. spray. As Lemma 5.4 shows, if $L: T M \rightarrow \mathbb{R}$ is a $G$ invariant Lagrangian, then $L$ is constant along the geodesics, that is along the integral curves of $S$. Consequently we have $d_{S} L=0$. Using Corollary 3.5 and the same argument that was used for Proposition 4.4 we can obtain the proof of the proposition.

\footnotetext{
${ }^{1}$ In [17] the terminology horizontal lift was used. However, in Finsler geometry, this terminology is widely used for a different object.
} 
We remark that the connection determined by a g.o. spray is not necessarily linear, therefore it is not true in general that the Finsler metrizability entail the Riemann metrizability as it was the case for the canonical spray of Lie groups. However, if the g.o. spray is quadratic then the associated connection is linear. Therefore we can use Szabó's theorem and similarly to Theorem 4.5 we can get the following

Theorem 5.6. A quadratic g.o. spray is invariant projectively Finsler metrizable if and only if it is invariant Riemann metrizable.

Remark. A different invariant metrizability concept of the $G / H$ structure is considered in [9] by S. Deng and Z. Hou where the $G / H$ structure is called invariant metrizable if there exists an invariant metric on it. The invariant metrizability (and projective metrizability) of a g.o. structure or g.o. spray is however more subtle, because in this case not only the $G / H$ homogeneous space, but also the geodesic structure is fixed and we want to metrize both. It may happen that the g.o. structure on a homogeneous space $G / H$ is not invariant metrizable but the $G / H$ structure it is. To illustrate this phenomenon, let us consider the following example.

Example. Let us consider $M=\mathbb{R}^{2}$ as the homogeneous space $\mathbb{R}^{2}=G / H$, where $G=$ $A S O(2)$ is the euclidean transformation group, and $H=S O(2)$ is the rotation subgroup. $H$ is the stabiliser of the point $o=(0,0) \in \mathbb{R}^{2}$. The matrix representation can be given by

$$
G=\left\{\left(\begin{array}{ccc}
\cos t-\sin t & x_{1} \\
\sin t & \cos t & x_{2} \\
0 & 0 & 1
\end{array}\right)\right\}, \quad H=\left\{\left(\begin{array}{ccc}
\cos t-\sin t & 0 \\
\sin t & \cos t & 0 \\
0 & 0 & 1
\end{array}\right)\right\},
$$

and the corresponding Lie algebras are

$$
\mathfrak{g}=\left\{\left(\begin{array}{ccc}
0 & -a_{3} & a_{1} \\
a_{3} & 0 & a_{2} \\
0 & 0 & 0
\end{array}\right)\right\}, \quad \mathfrak{h}=\left\{\left(\begin{array}{ccc}
0 & -a_{3} & 0 \\
a_{3} & 0 & 0 \\
0 & 0 & 0
\end{array}\right)\right\} .
$$

We have the identification $G / H \simeq \mathbb{R}^{2}$, and the projection map is given by

$$
\pi: G \rightarrow G / H \simeq \mathbb{R}^{2}, \quad\left(\begin{array}{ccc}
\cos t-\sin t & x_{1} \\
\sin t & \cos t & x_{2} \\
0 & 0 & 1
\end{array}\right) \stackrel{\pi}{\rightarrow}\left(\begin{array}{c}
x_{1} \\
x_{2} \\
1
\end{array}\right) \sim\left(x_{1}, x_{2}\right) .
$$

Using the identification " $\sim$ " introduced in (5.4), the action of $G$ can be interpreted as a matrix multiplication: $\lambda_{g} \widehat{x}=g \cdot \widehat{x}$. Since the homogeneous lift for this $G / H$ structure must be a rotation-invariant map, homogeneous of degree 1 , it is of the form

$$
\sigma_{\kappa}: T_{o} \mathbb{R}^{2} \rightarrow \mathfrak{g}, \quad\left(v_{1}, v_{2}\right) \stackrel{\sigma_{\kappa}}{\longrightarrow}\left(\begin{array}{ccc}
0 & -\kappa \sqrt{v_{1}^{2}+v_{2}^{2}} & v_{1} \\
\kappa \sqrt{v_{1}^{2}+v_{2}^{2}} & 0 & v_{2} \\
0 & 0 & 0
\end{array}\right),
$$

where $\kappa \in \mathbb{R}$ is an arbitrary (but fixed) constant. The geodesic equation corresponding to the homogeneous lift (5.5) can be given as follows: a curve $t \rightarrow \gamma(t)$ is a geodesic if and only if

$$
\ddot{\gamma}=\sigma_{\kappa}(\dot{\gamma}) \dot{\gamma}
$$

Indeed, let $\gamma(t)$ be the geodesic in the direction $v \in T_{o} M$ and let $X=\sigma_{\kappa}(v)$ be the geodesic vector corresponding to $v$. Using the matrix representation we get $\gamma(t)=e^{t \sigma_{\kappa}(v)} o$ and therefore for its second derivative we can find $\ddot{\gamma}(t)=\sigma_{\kappa}(v) \sigma_{\kappa}(v) e^{t \sigma_{\kappa}(v)} O=\sigma_{\kappa}(v) \dot{\gamma}(t)$. Since $v=\dot{\gamma}(0)$ and the tangent vectors are invariant to translations, we can obtain (5.6). The system of differential equations of geodesics is

$$
\left\{\begin{array}{l}
\ddot{x}_{1}=-\kappa \sqrt{\dot{x}_{1}^{2}+\dot{x}_{2}^{2}} \dot{x}_{2}, \\
\ddot{x}_{2}=\kappa \sqrt{\dot{x}_{1}^{2}+\dot{x}_{2}^{2}} \dot{x}_{1} .
\end{array}\right.
$$


If $\kappa=0$, then the curvature tensor is identically zero and the geodesics are straight lines. The corresponding g.o. structure is metrizable: the usual euclidean metric $\left(g_{i j}=\delta_{i j}\right)$ metricises it. If $\kappa \neq 0$, then the geodesics are non-reversible (that is $\gamma_{p,-v}(t) \neq \gamma_{p, v}(-t)$ ) and the curvature tensor is non-zero:

$$
R_{(x, y)}^{\kappa}\left(\frac{\partial}{\partial x_{1}}, \frac{\partial}{\partial x_{2}}\right)=-\kappa^{2} y_{2} \frac{\partial}{\partial y_{1}}+\kappa^{2} y_{1} \frac{\partial}{\partial y_{2}} .
$$

It is easy to show, using the metrizability criteria of [5] or [15], that the corresponding g.o. structure is not metrizable. Therefore it is not invariant metrizable as well.

This examples illustrates clearly the difference between the concept of the metrizability of the $G / H$ structure and the metrizability of the g.o. structure: the homogeneous space $\mathbb{R}^{2}=A S O(2) / S O(2)$ is invariant metrizable (for example an invariant metric is given by the standard euclidean metric), however the g.o. structure determined by the homogeneous lift (5.5) is not, unless $\kappa=0$.

\section{Acknowledgments}

We appreciate all the comments from an anonymous reviewer, especially those related to Corollary 3.5. This work has been supported by the EU FET FP7 BIOMICS project, contract number CNECT-318202.

\section{References}

[1] Anderson, I., Thompson, G.: The inverse problem of the calculus of variations for ordinary differential equations, Mem. Am. Math. Soc. 98, No.473, 110 p. (1992).

[2] Arnold, V.I.: Sur la géométrie différentielle des groupes de Lie de dimension infinie et ses applications á l'hydrodynamique des fluides parfaites, Ann. Inst. Fourier, 16 (1960), 319-361.

[3] Bryant, R.L., Dunajski, M., Eastwood, M.: Metrisability of two-dimensional projective structures, J. Differential Geom. 83, Number 3 (2009), 465-500.

[4] Bucataru, I., Muzsnay, Z.: Projective and Finsler metrizability for sprays: parameterization-rigidity of the geodesics, Int. J. Math., 23, no. 9, (2012) 1250099.

[5] Bucataru, I., Muzsnay, Z.: Metrizable isotropic second-order differential equations and Hilbert's fourth problem, J. Aust. Math. Soc, 97, 01 (2014), 27-47.

[6] Chern, S.S.: Finsler geometry is just Riemannian geometry without the quadratic restriction, Notices AMS, 43 (1996), 959-63.

[7] Crampin, M., Mestdag, T.: The inverse problem for invariant Lagrangians on a Lie group, J. Lie Theory 18: (2) (2008), 471-502.

[8] Crampin, M., Mestdag, T., Saunders, D.J.: Hilbert forms for a Finsler metrizable projective class of sprays, Diff. Geom. Appl. 31, 1, (2013), 63-79.

[9] Deng, S., Hou, Z.: Invariant Finsler metrics on homogeneous manifolds, J. Phys. A: Math. Gen. 37, (2004), 8245-8253.

[10] Ghanam, R., Hindeleh, F., Thompson, G.: Bi-invariant and noninvariant metrics on Lie groups J. Math Phys; Vol. 48 Issue 10, p.102903. 
[11] Ghanam, R., Thompson, G., Miller, E.J.: Variationality of Four-Dimensional Lie Group Connections, Journal of Lie Theory, Vol. 14 (2004) 395-425.

[12] Grifone, J., Muzsnay, Z.: Variational Principles For Second-Order Differential Equations, World Scientific, Singapore, 2000.

[13] Matveev, V.S.: Geodesically equivalent metrics in general relativity, J.Geom.Phys. $62,(2012), 675-691$.

[14] Mestdag, T.: Relative equilibra of invariant Lagrangian systems on a Lie Group, Geometric Methods in Mechanics and Field Theory. Gent: Academia Press, (2007), 91-114.

[15] Muzsnay, Z.: The Euler-Lagrange PDE and Finsler metrizability, Houston Journal of Mathematics, 32 no. 1, (2006) pp. 79-98.

[16] Muzsnay, Z.: An invariant variational principle for canonical flows on Lie groups, J. Math. Phys. 46 (2005), no. 11, 112902, pp. 11.

[17] Muzsnay, Z., Nagy, P.T.: Invariant Shen connections and geodesic orbit spaces , Period. Math. Hungar. 51 (2005), no. 1, 37-51.

[18] Muzsnay, Z., Thompson, G.: Inverse problem of the calculus of variations on Lie groups, Diff. Geom. Appl. 23 (2005), no. 3, 257-281.

[19] Rapcsák, A.: Über die bahntreuen Abbildungen metrischer Räume, Publ. Math. Debrecen 8 (1961), 285-290.

[20] Szabó, Z.: Positive definite Berwald spaces, Tensor, New Ser., 35 (1981), 25-39.

[21] Szilasi, J., Vattamány Sz.: On the Finsler-metrizabilities of spray manifolds, Period. Math. Hun. Vol. 44 (1), (2002), pp. 81-100.

[22] Strugar, I., Thompson, G.: The Inverse Problem for the Canonical Lie Group Connection in Dimension Five, Houston J. Math., Vol. 35, (2), (2009), 373-409.

[23] Thompson, G.: Variational connections on Lie groups, Diff. Geom. Appl., 18 (2003), $255-270$.

Ioan Bucataru

Faculty of Mathematics, Alexandru Ioan Cuza University

700506, Bulevardul Carol I 11, Iaşi, Romania

E-mail: bucataru@uaic.ro

Tamás Milkovszki

Institute of Mathematics, University of Debrecen,

H-4032 Debrecen, Egyetem tér 1, Hungary

E-mail: milkovszki@science.unideb.hu

Zoltán Muzsnay

Institute of Mathematics, University of Debrecen,

H-4032 Debrecen, Egyetem tér 1, Hungary

E-mail: muzsnay@science.unideb.hu 